\title{
Troxerutin protects against DHT-induced polycystic ovary syndrome in rats
}

\section{Zixuan Gao}

xuzhou medical university

Xiaochen Ma

xuzhou medical university

Jing Liu

Xuzhou Medical University

Yuhang Ge

Xuzhou Medical University

Lei Wang

Xuzhou Medical University

Ping Fu

Xuzhou Medical University

Zhian Liu

Xuzhou Medical University

Ruiqin Yao

Xuzhou Medical University

Xiaonan Yan ( $\nabla$ yxn_central@126.com)

Xuzhou City Centre Hospital https://orcid.org/0000-0001-5383-3960

\section{Research}

Keywords: PCOS, Troxerutin, GnRH, LH, Neurotransmitter

Posted Date: July 31st, 2020

DOI: https://doi.org/10.21203/rs.3.rs-16993/v2

License: (c) (i) This work is licensed under a Creative Commons Attribution 4.0 International License.

Read Full License

Version of Record: A version of this preprint was published on September 13th, 2020. See the published version at https://doi.org/10.1186/s13048-020-00701-z. 


\section{Abstract}

The exact pathogenesis of polycystic ovary syndrome (PCOS), the most common neuroendocrine disorder in women of reproductive age, has not been fully elucidated. Recent studies suggested that chronic inflammation and neurotransmitter disorder involved in the progress of PCOS. Troxerutin, a natural flavonoid, was reported to possess neuroprotective effect in several disease models by inhibiting inflammation or enhancing neurotrophic factor. In this study, we investigated the possible protective effect and mechanism of troxerutin in a dihydrotestosterone (DHT)-induced rat model of PCOS. The PCOS rat models were treated with troxerutin at a dose of $150 \mathrm{mg} / \mathrm{kg}$ or $300 \mathrm{mg} / \mathrm{kg}$ for up to 4 weeks.

Results showed that $300 \mathrm{mg} / \mathrm{kg}$ troxerutin significantly decreased the body weight gain and improved the pathological changes of ovary induced by DHT. Meanwhile, the elevated gonadotrophin-releasing hormone $(\mathrm{GnRH})$, gonadotrophin and testosterone in the serum of PCOS rats were reduced with the treatment of troxerutin. The expression of kisspeptin and NKB in arcuate nucleus and their receptors kiss $1 r$ and NK3 $r$ in GnRH positive neurons of median eminence were markedly decreased in troxerutintreated rats. Of note, the $\mathrm{GnRH}$ inhibitory regulator GABA and stimulatory regulator glutamate were also restored to the normal level by troxerutin. The present study indicated that troxerutin may exhibit a protective effect in PCOS rat model via regulating neurotransmitter release.

\section{Introduction}

Polycystic ovary syndrome (PCOS) is a reproductive endocrinopathy with the prevalence estimated to be $6 \% \sim 20 \%$ (depending on the different diagnostic criteria used), making it the most common endocrine condition in women of reproductive age [1]. The diagnostic features of PCOS include androgen excess, ovulatory dysfunction and polycystic ovaries[1]. Obesity is present in $30-60 \%$ of PCOS patients, depending on the country of origin[2,3]. The most consistent biochemical abnormality in women with PCOS is hyper-secretion of androgen, elevated serum luteinizing hormone (LH) levels and low to normal serum follicle stimulating hormone (FSH) levels $[4,5]$. Animal models that reflect PCOS features are crucial resources to investigate this syndrome. A chronically 5a-dihydrotestosterone (DHT)-treated rat model closely mimics the human PCOS phenotype and is a suitable model for investigations about PCOS.

The hypothalamus-pituitary-gonad (HPG) axis plays a central role in the exquisite neuroendocrine regulation of reproduction. Hypothalamic secretion of gonadotrophin-releasing hormone (GnRH), has been robustly established as the key pathway that controls reproductive function[6]. Axons of GnRH neurons protrude into the median eminence and release $\mathrm{GnRH}$, which via hypophyseal portal system, enters anterior pituitary. Anterior pituitary, the primary target of $\mathrm{GnRH}$, responds to stimulation by increasing the secretion of $\mathrm{LH}$ and $\mathrm{FSH}$, which in turn lead to steroid production from the ovaries and stimulate follicle genesis and ovulation[7]. Thus, $\mathrm{GnRH}$ is known as the master hormone and provides the final common output of the network that regulates reproductive function. 
$\mathrm{GnRH}$ is influenced by extrinsic factors such as neurotransmitters and neuropeptides, any alteration of the $\mathrm{GnRH}$ regulatory neurotransmitters, such as inhibitory gamma-aminobutyric acid( GABA) and stimulatory glutamate(Glu), may result in reproductive endocrine dysfunction[8]. Actually, the chronic anovulation characteristic of PCOS is attributed to increased central GnRH drive and resulting gonadotrophin aberrations, which likely result from the cumulative effect of altered GnRH stimulatory and inhibitory neurotransmitter in hypothalamus and pituitary gland[9]. Kisspeptin and subsequently discovered neurkinin $\mathrm{B}(\mathrm{NKB})$, two novel $\mathrm{GnRH}$ regulatory neuropeptides proved to be essential for normal $\mathrm{GnRH}$ secretion in humans, have come under intense spotlight in the last decade[10,11]. The related discovery of kisspeptin-/ neurokinin B-/ dynorphin-(KNDy) pathway has further strengthened the understanding of $\mathrm{GnRH}$ secretion modulation[12]. KNDy neurons residing in the arcuate nucleus region of rodents co-express kisspeptin, NKB and DYN $[12,13]$. NKB and kisspeptin derived from KNDy stimulate the release of $\mathrm{GnRH}$ by binding Kisspeptin 1 receptor (Kiss1r)and neurokinin 3 receptor(NK3r) expressed on GnRH neurons[14, 15].

Troxerutin, a trihydroxyethylated derivative of the naturally occurring plant bioflavonoid rutin, also called vitamin P4. It has been widely identified in plants consumed as part of our daily diet, such as tea, coffee, cereals, and a variety of fruits and vegetables, and exerts multifarious biological activities, such as antioxidant and anti-inflammatory effects and exerts beneficial effects on animal models of different central nervous system diseases $[16,17]$. Troxertuin attenuated cognitive deficit and oxidative stress in mouse brain through decreasing reactive oxygen species[18],inhibited hippocampal neuron apoptosis in a rat model of Alzheimer's disease[19]. Troxerutin inhibited cyclin-dependent kinase 1 expression, enhanced type 1 protein phosphatase a dephosphorylation and abolished MEK/ERK1/2/C/EBP $\beta$ activation, which subsequently reversed the memory impairment in the domoic acid-treated mice[20], and exhibited neuroprotective potential in 6-OHDA rat model of Parkinson's disease through mitigation of apoptosis ,astrogliosis and oxidative stress [21], prevented the harmful effects of maternal high-fat diet on their offspring through inhibition of pro-inflammatory cytokines and elevation of brain-derived neurotrophic factor levels[22], significantly promoted nerve growth factor (NGF) mRNA expression and attenuated cognitive impairment and brain oxidative stress through the activation of NGF/TrkA signaling pathway in D-gal-injected mice[23]. However, there was no evidence that troxerutin had any effect on IGF-1 so far.Since the hypothalamus plays an important role in reproduction, we supposed that troxerutin may have a protective effect against reproductive neuroendocrine dysfunction in PCOS. This study aimed to address this issue and preliminarily investigate the potential mechanism underlying its action.

\section{Materials And Methods}

\section{Animals and troxerutin administration}

Postnatal 14 days female SD rats were purchased from the Breeder center of Rodents (Jinan, China).The rats were housed under controlled lighting (12h light, $12 \mathrm{~h}$ darkness, lights were turned on at 08:00 and turned off at 20:00) and temperature $\left(24^{\circ} \mathrm{C}\right)$ with free access to food and water for a week of acclimatization. All experimental protocols used in this study were approved by the Animal Care and 
Ethical Committee of Xuzhou medical University. Postnatal 21 days rats weighing approximately 50-55g, were randomly divided into four groups: sham group (each rat was implanted with a tube without DHT), PCOS group (each rat was implanted with a silastic tube filled with 5a-DHT at dose of $7.5 \mathrm{mg}$ under anesthesia), PCOS $+150 \mathrm{mg} / \mathrm{kg}$ troxerutin group and PCOS $+300 \mathrm{mg} / \mathrm{kg}$ troxerutin group (PCOS rat with intraperitoneal injection of $150 \mathrm{mg} / \mathrm{kg}$ or $300 \mathrm{mg} / \mathrm{kg}$ troxerutin daily during the 4 weeks after implantation). Due to the high water-solubility of troxerutin, the gastrointestinal system can absorb it well without toxicity. Troxerutin interacts with tRNA by external binding manner with low binding affinity, and also binds with DNA through groove binding manner. Sham and PCOS groups were injected daily with equivalent $0.9 \%$ saline which was used to dissolve troxerutin. DHT was procured from sigma, St. Louis, Mo and troxerutin (purity >95\%) was from Baoji Fangsheng Biotechnology Co., Ltd, Baoji, China. The rats were housed individually after surgery.

\section{Blood and tissue sampling}

At the terminal of the study, rats were decapitated and trunk blood was collected and centrifuged at 3000 rpm for 15 minutes. Plasma was separated and stored at $-80^{\circ} \mathrm{C}$ until analyzed for biochemical and hormonal analysis. Ovaries were cleaned in saline and managed fat free. Ovaries were fixed in $10 \%$ buffered formalin for 48 hours and paraffin-embedded. Paraffin-embedded tissue sections were dewaxed, sectioned $(6 \mu \mathrm{m})$ and stained with hematoxylin and eosin (H\&E). Numbers of follicles were classified and counted in every $40^{\text {th }}$ ovarian section for 10 ovaries per group. Follicles were counted according to the following definition. Primordial follicle was surrounded by a single layer of squamous granulosa cells. Primary follicle contained a single layer of cuboidal granulosa cells. Secondary follicle contained two or more layers of granusola cells, but no antral space in the granulosa layer. Antral follicle displayed multi-layers of granusola cells with the presence of follicular antrum.

\section{Vagina smears and estrous cycles}

Vaginal smears were taken daily at 09:00am from the $18^{\text {th }}$ to the $28^{\text {th }}$ day after the first day of DHT treatment,which were the terminal ten days of the study. Microscopic analysis (BX41, Olympus) was carried out to determine the stage of the estrous cycle by the predominant cell types in vaginal smears following methylene blue staining(Solarbio, Beijing). Proestrus consists of enlarged,round,nucleated epithelical cells; estrus consists of a large number of cornified squamous epithelical cells; metestrus consists of two kinds of cells: epithelial cells and leukocytes with approximately same amount; and diestrus is characterized by a small amount of cells with the predominance of leukocytes.

\section{Body weight, biochemical and western blot analysis}

Body weight was measured every week after implantation for a total of 4 weeks.

The serum concentration of LH, FSH and testosterone were measured via Enzyme Linked Immuno Sorbent Assay (ELISA) with the help of commercial kits (ELISA kit, CUSABIO, Inc, Wuhan, China)and the 
procedure was followed as given in the kit catalog. The concentration of LH, FSH and testosterone was estimated by the standard curve.

To identify the hypothalamic GnRH status, hypothalamus was dissected out. The rat brain was carefully taken out and temporarily put on ice with the ventral surface upward. Thalamus is the ellipsoidal part at the center of the ventral brain. With the middle of tuber cinereum and optic chiasma as the center, prechiasmal border as the anterior edge, postmammillary border as the posterior edge, hypothalamus $(4 \times 4 \times 2 \mathrm{~mm})$ was carefully dissected out.Hypothalamus tissue were homogenized to obtain protein samples. Then the protein $(25 \mu \mathrm{g})$ was separated by SDS-PAGE and transferred to membranes. The membranes were incubated with mouse monoclonal anti-GnRH antibody (1:1000, MAB5456-C, Millipore, Billerica, MA) and mouse anti-GAPDH antibody (1:20000, Proteintech, Chicago, USA). After being washed with TBST three times, the membranes were incubated with IRDye-labeled secondary antibodies in TBST

for $2 \mathrm{~h}$. The bands on the membrane were scanned with an Odyssey infrared scanner (LI-COR Biosciences, Lincoln, NE, USA) and the density of the bands was analyzed with Image J software.

\section{Chromatographic analysis}

Chromatographic analysis was carried out to evaluate the levels of two kinds of $\mathrm{GnRH}$ regulatory neurotransmitters GABA and glutamate in the brain/pituitary. Weigh the brain and add in the mix of methanol and water $(1: 1)$ at the ratio of $100 \mathrm{mg} / \mathrm{ml}$ to prepare homogenate. The brain homogenate was centrifuged at $12000 \mathrm{rpm}$ for $30 \mathrm{~min}$ at $4^{\circ} \mathrm{C}$. The mix of supernate and $80 \mu \mathrm{L}$ acetonitrile were centrifuged at $12000 \mathrm{rpm}$ for $30 \mathrm{~min}$ at $4^{\circ} \mathrm{C}$. The new supernate was derivatized, mixed with $80 \mu \mathrm{L}$ borate saline buffer, $160 \mu \mathrm{L}$ water and $80 \mu \mathrm{L} \mathrm{F-moc}$, filtered by $0.45 \mu \mathrm{m}$ filtration membrane and immersed in $40^{\circ} \mathrm{C}$ bath for 5 minutes. Then, the samples were used for the measurement of GABA and glutamate using chromatographic analysis.All standard biomarkers used for identification purpose in chromatographic studies were procured from Zhongke Co, Ltd, Beijing, China. High-performance liquid chromatography \HPLC『was performed on a Waters e2695 system(Waters, USA)equipped with a hypersil ODS column(Elite, Dalian, China). The mobile phase $A$ was a mix of natrium aceticum(pH4.8), water and tetrahydrofuran with the ratio of 410:85:5, and mobile phase $B$ was pure acetonitrile. Flow rate was $1 \mathrm{ml} / \mathrm{min}$. The column temperature was set at $30^{\circ} \mathrm{C}$. Samples were detected the wavelength of $265 \mathrm{~nm}$. Injection volume was $20 \mu \mathrm{L}$.

\section{Immunofluorescent staining}

After intracardiac perfusion with normal saline followed by fixation with $4 \%$ cold paraformaldehyde (PFA), the rat brains were separated and postfixed in $4 \%$ PFA for $6 \mathrm{~h}$ at $4{ }^{\circ} \mathrm{C}$. Then the brains were incubated in $30 \%$ sucrose- $100 \mathrm{mM}$ sodium phosphate buffer $(\mathrm{pH} 7.4)$ for $48 \mathrm{~h}$ at $4{ }^{\circ} \mathrm{C}$. Serial coronal sections $(20 \mu \mathrm{m})$ were made from the bregma anterior-posterior $-2.0 \mathrm{~mm}$ to $-3.30 \mathrm{~mm}$. Totally 60 sections were collected per brain. At least three sections per brain were selected for NKB, Kisspeptin, $\mathrm{GnRH} / \mathrm{NK} 3 \mathrm{R}$ and $\mathrm{GnRH} / \mathrm{Kiss} 1 \mathrm{r}$ immunofluorescent staining, respectively. 
For immunofluorescence, the primary antibodies rabbit anti-NKB (1:1000, Novus biologicals, NB300-201), rabbit anti-kisspeptin (1:1000, H-048-56, Phoenix Pharmaceuticals), rabbit anti-Kiss-1r (1:500, AKR-001, Alomone Labs), rabbit anti-NK3r(1:500, abx217136, Abbexa) and mouse anti-GnRH (1:1000, MAB5456-C, Millipore, Billerica, MA) were used. After incubating with the antibodies for $24 \mathrm{~h}$ at $4{ }^{\circ} \mathrm{C}$, the sections were washed with PBS and then were treated with goat anti-mouse $\operatorname{lgG}(\mathrm{H}+\mathrm{L})$ Alexa Fluor ${ }^{\circledR} 555$ or 488 conjugated or goat anti-rabbit IgG $(\mathrm{H}+\mathrm{L})$ Alexa Fluor ${ }^{\circledR} 488$ (Invitrogen, Eugene, OR, USA) secondary antibodies. According to the manufacturer's instructions, DAPI (Beyotime Biotechnology, Shanghai, China) was used to label nucleus. For negative controls, sections were incubated with PBS instead of the primary antibodies. Fluorescence images were captured using a Zeiss Axioskop 40 microscope(Carl Zeiss, Oberkochen, Germany). Image Pro-Plus 6.0 software was used for semi-quantitative measurement of immunofluorescent density. Values (three slides for each brain) of integral optical density (IOD) in individual cells represented the quantity of objective protein and were calculated using the following equation: $\Sigma$ IOD/ $\Sigma$ DAPI.

\section{Statistical analysis}

All statistical analyses were performed with SPSS software (version 16.0), and the data were analyzed using the one-way Analysis of Variance (ANOVA). The data were expressed as the mean \pm s.e.m. Statistical significance was set at $\mathrm{P}<0.05$ for all tests.

\section{Results}

\section{Troxerutin reduced body weight, improved abnormal ovarian morphology and function in PCOS rats}

We first investigated whether troxerutin administration has any influence on body weight in DHT-induced PCOS rat models. The time course of troxerutin administration and evaluation of phenotypes were illustrated (Figure 1). Body weight for individual animal was weighed weekly after DHT implantation for up to 4 weeks. Body weight at baseline and 1st week showed no significant difference among all groups. DHT-treated rats showed significantly higher body weight at $2 \mathrm{nd}(P<0.01), 3 \mathrm{rd}(P<0.01)$ and 4 th $(P<0.001)$ week than the age-matched sham rats which denoted normal body weight, indicating that PCOS group have gained more body weight, hence a PCOS-like over weight feature. In comparison to PCOS group, treatment with troxerutin $150 \mathrm{mg} / \mathrm{kg}$ did not induce any significant changes. Nevertheless, the body weight of PCOS $+300 \mathrm{mg} / \mathrm{kg}$ troxerutin group were markedly reduced as compared to that of PCOS group at 2 nd $(P<0.05), 3 \mathrm{rd}(P<0.01)$ and 4 th $(P<0.05)$ week, suggesting that troxerutin alleviated increased body weight in PCOS. Since ovarian histological change is another feature of PCOS, we also investigated whether troxerutin improved histological structure in rat ovaries. As demonstrated in figure $1 \mathrm{~b}$ and $1 \mathrm{c}$, normal ovarian histological features were observed in sham sections, which generally exhibited corpora lutea and follicles in various stages of development. In ovarian sections of PCOS rats, microscopic examination confirmed the absence of corpora lutea and the presence of cystic follicles, which were characterized by a large fluid-filled structure with an attenuated granulosa cell layer and a thickened theca internal cell layer. We furthered calculated the percentage of follicles of total and the results 
showed that PCOS rats exhibited significantly increased primordial and decreased primary follicles as compared to sham rats $(P<0.001 ; P<0.001)$, and troxerutin $300 \mathrm{mg} / \mathrm{kg}$ inhibited the markedly low percentage of primary follicles in PCOS rats $(P<0.05)$. As compared to the multi-cystic follicles in ovaries of PCOS rats, the number of cystic follicles in troxerutin groups showed tendency toward decreasing. As figure 1.d demonstrated, sham group showed normal and stable estrous cycle, DHT induced cycle disruption in PCOS group, troxerutin administration $300 \mathrm{mg} / \mathrm{kg}$ in PCOS rat models prevented and substantially reversed the impaired estrous cycle. These findings suggest that troxerutin is a positive regulator of increased body weight,abnormal ovarian histological structure and ovary function in PCOS rats.

\section{Troxerutin reversed the abnormal serum levels of gonadotrophin and testosterone in PCOS rats}

Serum LH, FSH and testosterone levels were measured at the fourth week by ELISA (Figure 2). PCOS rats showed a significant increase in serum LH and testosterone levels compared to sham rats $(P<0.001$; $\mathrm{P}<0.05)$, which was in accordance with the characteristic $\mathrm{LH}$ and testosterone elevation in patients with PCOS. Though there was no significant difference in FSH levels among all four groups, administration of troxerutin to rats for 4 weeks significantly reversed the elevated serum $\mathrm{LH}(300 \mathrm{mg} / \mathrm{kg} ; \mathrm{P}<0.01)$ and testosterone levels (both $150 \mathrm{mg} / \mathrm{kg}$ and $300 \mathrm{mg} / \mathrm{kg} ; P<0.05$ ), suggesting that troxerutin has a beneficial effect on the aberrant gonadotrophin and testosterone in PCOS rats.

\section{Troxerutin inhibited the elevated GnRH levels in PCOS rats}

The origin of $\mathrm{LH}$ and FSH alteration often times lies at hypothalamic GnRH level, which plays a pivotal role in stimulating pituitary release of gonadotrophin, thus we further detected the serum and hypothalamic-pituitary status of GnRH. As shown in figure 3, the western blotting results showed increased GnRH in hypothalamic-pituitary of the PCOS rats $(P<0.01)$, however, troxerutin $300 \mathrm{mg} / \mathrm{kg}$ markedly decreased the level of GnRH compared to the PCOS rats $(P<0.05$; Figure $3 b)$.

\section{Troxerutin altered GnRH regulatory neurotransmitters in the hypothalamus of PCOS rats.}

GnRH could be influenced by regulatory neurotransmitters such as the major inhibitory GABA and stimulatory Glu. As depicted in figure 4, neurotransmitter levels in the hypothalamus showed significant difference among certain groups. To be specific, PCOS rats exhibited markedly low GABA $(P<0.01)$ and high Glu $(P<0.01)$ compared to sham rats. Although no significant difference was observed between troxerutin $150 \mathrm{~kg} / \mathrm{mg}$ group and PCOS group, administration of troxerutin $300 \mathrm{~kg} / \mathrm{mg}$ to rats for 4 weeks successfully reversed the notably low GABA $(P<0.05)$ and high Glu $(P<0.01)$ levels in the hypothalamus compared to PCOS group.

\section{Troxerutin reduced the expression of Kisspepetin1/Kiss1 $r$ and Neurokinin B/NK3r in the hypothalamus}

Kisspeptin and NKB secreted from the KNDy neurons in ARC, are considered to be novel GnRH stimulatory neurotransmitters and stimulate expression of kisspeptin receptor (kiss $1 r$ ) and neurokinin B receptor (NK3r) of GnRH neurons in the median eminence (ME). Here, we carried out immunofluorescence 
staining to observe whether troxerutin administration alters the expression of kisspeptin/ kiss1r and NKB/ NK3r in their respective regions (Figure 5 and Figure 6). The IOD of positive cells in the ARC were markedly increased in PCOS rats compared to sham rats $(P<0.001 ; P<0.001)$, troxerutin $300 \mathrm{mg} / \mathrm{kg}$ treatment significantly reversed the increase of kisspeptin and NKB IOD induced by DHT $(P<0.05 ; P<0.01)$. Meanwhile, the changes of the expression of kiss1r and NK3r in ME were also observed. The numbers of $\mathrm{GnRH} / \mathrm{kissr} 1 / \mathrm{NK} 3 \mathrm{r}$ positive cells increased in the PCOS group compared to the sham group $(\mathrm{P}<0.001$; $P<0.01 ; P<0.01)$, however, they were significantly decreased in the $300 \mathrm{mg} / \mathrm{kg}$ troxerutin-treated rat $(P<0.01 ; P<0.05 ; P<0.05)$ respectively.

\section{Discussion}

PCOS is the most common endocrine condition affecting approximately $20 \%$ of reproductive -aged women. Though some ovulation induction agents, such as letrozole, clomiphene citrate and metformin, have been used to improve reproductive outcomes in clinic, the side effects of these drugs should not be ignored[24, 25]. Thus, numerous studies attempted to guide the development of new and effective therapies for PCOS. Recently, treatment based on traditional Chinese medicine and food ingredients, such as AF extract, soy isoflavones and crocetin, provided a novel therapeutic way for PCOS [26-28]. All these observations suggested rooms for improvement in PCOS therapies. Possession of beneficial effects on animal models of different central nervous system diseases made troxerutin, a rutin derivative, an attractive therapeutic method for us to investigate its possible effect on PCOS. Specifically, troxerutin inhibited cyclin-dependent kinase 1 expression, enhanced type 1 protein phosphatase a dephosphorylation and abolished MEK/ERK1/2/C/EBP $\beta$ activation, which subsequently reversed the memory impairment in the Domoic acid-treated mice[20]. The neuroprotective potential of troxerutin in 6OHDA rat model of Parkinson's disease was through mitigation of apoptosis ,astrogliosis, oxidative stress and part of its effect was dependent on PI3K/ER $\beta$ signaling[21]. Troxerutin and cerebroprotein hydrolysate injection acted as a neuroprotective agent against cerebral ischemia/reperfusion injury via anti-inflammation, anti-apoptosis and blood-brain barrier maintenance[29]. In the present study, we evaluated the possible effect of troxerutin for up to four weeks in a DHT-induced PCOS rat model, and two important findings were revealed. First, administering troxerutin $300 \mathrm{mg} / \mathrm{kg}$ exerted a beneficial effect of reducing body weight, the elevated levels of $\mathrm{LH}$, testosterone and GnRH in PCOS rats and its effect was superior to troxerutin $150 \mathrm{mg} / \mathrm{kg}$. Second, the potential mechanism behind the observed effect on increased body weight, polycystic ovaries, impaired estrous cycle and endocrine aberration involved altering GABA, glutamate, kisspeptin/kiss1 $r$ and NKB/NK3r in the hypothalamic pituitary region. To the best of our knowledge, this is the first study to investigate the possible effect of troxerutin on PCOS using a rat model and the potential mechanism of its protective effect may be via regulating GABA, glutamate, kisspeptin/kiss $1 r$ and NKB/NK3r in the brain/pituitary.

GnRH pulses stimulate the synthesis and secretion of LH and FSH from anterior pituitary. It is well known that although produced in the same cell named gonadotroph, $\mathrm{LH}$ and FSH synthesis is regulated by different frequency of GnRH pulses, with LH favored by fast pulse frequencies( $>1$ pulse per hour) and FSH favored by slow pulse frequencies( $<1$ pulse per 2-3 hour). As for PCOS, a neuroendocrine hallmark is 
persistent and rapid GnRH pulses, which favor pituitary synthesis of $\mathrm{LH}$ and contribute to the increased LH levels[30]. LH is a stimulus for androgen synthesis, so the increased LH drive in turn caused the elevated androgens [31, 32]. Therefore, in terms of biochemical indicators, the most important and characteristic abnormality referring to PCOS is elevated LH, subsequently elevated testosterone and low to normal FSH levels in serum[4,33]. Consistent with previous studies, PCOS rats displayed similar biochemical abnormality including markedly increased LH, concomitantly increased testosterone levels and non-significant FSH levels as compared to sham rats. Next, we observed the serum LH, testosterone and FSH levels in PCOS and troxerutin groups in order to determine whether troxerutin treatment has any therapeutic effect on PCOS. As the results revealed, troxerutin treatment indeed inhibited the elevated LH and testosterone levels without significant influence of FSH level. In addition, we further estimated the hypothalamic status of $\mathrm{GnRH}$, which was significantly increased in PCOS rats. However, there was a significant troxerutin-caused ( $300 \mathrm{mg} / \mathrm{kg}$ ) decline in hypothalamic $\mathrm{GnRH}$. In regard to the serum hormone levels in rats, many previous studies have reported that LH were generally in the range of 1.5 3 $\mathrm{mIU} / \mathrm{ml}[34,35]$, FSH levels varied from $8 \mathrm{mlU} / \mathrm{ml}$ to $20 \mathrm{mlU} / \mathrm{ml}[26,36]$ and testosterone varied from 0.2 to $10 \mathrm{ng} / \mathrm{ml}[34,36-39]$, which were approximately consistent with the corresponding results in the present study. Conclusively, troxerutin treatment $300 \mathrm{~kg} / \mathrm{mg}$ to rats for up to 4 weeks inhibited the hyperactive $\mathrm{GnRH} / \mathrm{LH}$ system in PCOS rats.

Compelling evidence provided the strong support for brain being the culprit in PCOS[40, 41]. The secretion of $\mathrm{GnRH}$ from the brain is itself regulated by numerous upstream factors, such regulators include GABA, norepinephrine, dopamine, serotonin and glutamate ,amongst others[42, 43]. While specific impairments in the brain were difficult to assess in humans, rat models greatly facilitated the identification of difference underpinning the hyperactive HPG axis in PCOS. Therefore, the representative and GnRH regulators of inhibitory GABA and stimulatory Glu were observed in the present study. In comparison to sham rats, PCOS rats showed a turnover of regulatory neurotransmitters with a significantly decreased GABA and increased Glu, which were both reversed by administering troxerutin $300 \mathrm{mg} / \mathrm{kg}$ to rats for up to 4 weeks. As far as the hypothalamic neurotransmitter levels were concerned, previous studies reported that hypothalamic GABA was in range of $12-20 \mu \mathrm{M} / \mathrm{g}$ and hypothalamic Glu was in range of $22-45 \mu \mathrm{M} / \mathrm{g}$ in rats ,each approximately consistent with our findings after unit conversion. To date, one arcuate nucleus population of particular recent interest has been the kisspeptin/neurokinin B/dynorphin expressing 'KNDy' neurons, which synthesized and released GnRH stimulatory neurotransmitters kisspeptin and NKB. Protrusions of GnRH neurons in the ME express their receptors kiss $1 r$ and NK3r. In the present study, troxerutin decreased the expression of kisspeptin 1 and NKB in the arcuate nucleus, and also the receptors kiss1r and NK3r in GnRH positive neurons of the ME.

\section{Declarations}

\section{Acknowledgement}

We would like to thank everyone who take part in this study. 


\section{Authors' Contributions}

Zixuan Gao and Xiaochen Ma performed the experiments, collected and analyzed the data and prepared the manuscript. Yuhang Ge, Lei Wang and Ping Fu performed the experiments and provided the data. Zhian Liu, Ruiqin Yao, Xiaonan Yan designed the study, provided financial support and edited the manuscript. All the authors approved the final approval of the manuscript.

\section{Funding}

This research was supported by the funds granted by the national natural science foundation of China(No. 81571405) to Xiaonan Yan, Jiangsu Province natural science foundation of China(No. BK20161169)to Xiaonan Yan, the starting foundation for talents of xuzhou Medical University (No. D2018007) to Zixuan Gao, the Jiangsu Shuangchuang program to Zixuan Gao, Natural science research projects of Colleges and Universities in Jiangsu province(No. 19KJB310021)to Zixuan Gao.

\section{Availability of data and materials}

All the data is contained in the manuscript.

\section{Ethics approval}

All animal experiments were approved by the Institutional Animal Care Committee of Xuzhou Medical University and in strict accordance with the Guidelines for the Care and Use of Laboratory Animals(National Research Council of People's Republic of China,2010)

\section{Consent for publication}

Not applicable.

\section{Competing interests}

There is no conflict of interest to declare. The funders had no role in the study design; in the collection, analyses or interpretation of data; in the manuscript and decision to publish the results.

\section{References}

1. Goodarzi, M. O.; Azziz, R., Diagnosis, epidemiology, and genetics of the polycystic ovary syndrome. Best practice \& research. Clinical endocrinology \& metabolism 2006, 20, (2), 193-205.

2. Hahn, S.; Tan, S.; Elsenbruch, S.; Quadbeck, B.; Herrmann, B. L.; Mann, K.; Janssen, O. E., Clinical and biochemical characterization of women with polycystic ovary syndrome in North Rhine-Westphalia. Horm Metab Res 2005, 37, (7), 438-444.

3. Carmina, E.; Legro, R. S.; Stamets, K.; Lowell, J.; Lobo, R. A., Difference in body weight between American and Italian women with polycystic ovary syndrome: influence of the diet. Hum Reprod 
2003, 18, (11), 2289-2293.

4. Zumoff, B.; Freeman, R.; Coupey, S.; Saenger, P.; Markowitz, M.; Kream, J., A chronobiologic abnormality in luteinizing hormone secretion in teenage girls with the polycystic-ovary syndrome. $N$ Engl J Med 1983, 309, (20), 1206-1209.

5. Gilling-Smith, C.; Story, H.; Rogers, V.; Franks, S., Evidence for a primary abnormality of thecal cell steroidogenesis in the polycystic ovary syndrome. Clin Endocrinol (Oxf) 1997, 47, (1), 93-99.

6. Tsutsumi, R.; Webster, N. J. G., GnRH pulsatility, the pituitary response and reproductive dysfunction. Endocr J 2009, 56, (6), 729-737.

7. Kaiser, U. B.; Conn, P. M.; Chin, W. W., Studies of gonadotropin-releasing hormone (GnRH) action using GnRH receptor-expressing pituitary cell lines. Endocr Rev 1997, 18, (1), 46-70.

8. Smith, M. J.; Jennes, L., Neural signals that regulate GnRH neurones directly during the oestrous cycle. Reproduction 2001, 122, (1), 1-10.

9. Chaudhari, N.; Dawalbhakta, M.; Nampoothiri, L., GnRH dysregulation in polycystic ovarian syndrome (PCOS) is a manifestation of an altered neurotransmitter profile. Reprod Biol Endocrinol 2018, 16, (1), 37-37.

10. de Roux, N.; Genin, E.; Carel, J.-C.; Matsuda, F.; Chaussain, J.-L.; Milgrom, E., Hypogonadotropic hypogonadism due to loss of function of the KiSS1-derived peptide receptor GPR54. Proc Natl Acad Sci U S A 2003, 100, (19), 10972-10976.

11. Topaloglu, A. K.; Reimann, F.; Guclu, M.; Yalin, A. S.; Kotan, L. D.; Porter, K. M.; Serin, A.; Mungan, N. O.; Cook, J. R.; Imamoglu, S.; Akalin, N. S.; Yuksel, B.; O'Rahilly, S.; Semple, R. K., TAC3 and TACR3 mutations in familial hypogonadotropic hypogonadism reveal a key role for Neurokinin $B$ in the central control of reproduction. Nature genetics 2009, 41, (3), 354-358.

12. Goodman, R. L.; Lehman, M. N.; Smith, J. T.; Coolen, L. M.; de Oliveira, C. V. R.; Jafarzadehshirazi, M. R.; Pereira, A.; Iqbal, J.; Caraty, A.; Ciofi, P.; Clarke, I. J., Kisspeptin neurons in the arcuate nucleus of the ewe express both dynorphin A and neurokinin B. Endocrinology 2007, 148, (12), 5752-5760.

13. Foradori, C. D.; Amstalden, M.; Goodman, R. L.; Lehman, M. N., Colocalisation of dynorphin a and neurokinin B immunoreactivity in the arcuate nucleus and median eminence of the sheep. $J$ Neuroendocrinol 2006, 18, (7), 534-541.

14. Messager, S.; Chatzidaki, E. E.; Ma, D.; Hendrick, A. G.; Zahn, D.; Dixon, J.; Thresher, R. R.; Malinge, I.; Lomet, D.; Carlton, M. B. L.; Colledge, W. H.; Caraty, A.; Aparicio, S. A. J. R., Kisspeptin directly stimulates gonadotropin-releasing hormone release via G protein-coupled receptor 54. Proc Natl Acad Sci U S A 2005, 102, (5), 1761-1766.

15. Krajewski, S. J.; Anderson, M. J.; Iles-Shih, L.; Chen, K. J.; Urbanski, H. F.; Rance, N. E., Morphologic evidence that neurokinin B modulates gonadotropin-releasing hormone secretion via neurokinin 3 receptors in the rat median eminence. J Comp Neuro/ 2005, 489, (3), 372-386.

16. Panat, N. A.; Maurya, D. K.; Ghaskadbi, S. S.; Sandur, S. K., Troxerutin, a plant flavonoid, protects cells against oxidative stress-induced cell death through radical scavenging mechanism. Food Chem 2016, 194, 32-45. 
17. Zhang, Z.-f.; Fan, S.-h.; Zheng, Y.-I.; Lu, J.; Wu, D.-m.; Shan, Q.; Hu, B., Troxerutin protects the mouse liver against oxidative stress-mediated injury induced by D-galactose. J Agric Food Chem 2009, 57, (17), 7731-7736.

18. Lu, J.; Wu, D.-m.; Zheng, Z.-h.; Zheng, Y.-I.; Hu, B.; Zhang, Z.-f., Troxerutin protects against high cholesterol-induced cognitive deficits in mice. Brain 2011, 134, (Pt 3), 783-797.

19. Farajdokht, F.; Amani, M.; Mirzaei Bavil, F.; Alihemmati, A.; Mohaddes, G.; Babri, S., Troxerutin protects hippocampal neurons against amyloid beta-induced oxidative stress and apoptosis. EXCLI J 2017, 16, 1081-1089.

20. Palomba, S.; Falbo, A.; Zullo, F., Management strategies for ovulation induction in women with polycystic ovary syndrome and known clomifene citrate resistance. Curr Opin Obstet Gyneco/2009, 21, (6), 465-473.

21. Misso, M. L.; Teede, H. J.; Hart, R.; Wong, J.; Rombauts, L.; Melder, A. M.; Norman, R. J.; Costello, M. F., Status of clomiphene citrate and metformin for infertility in PCOS. Trends Endocrinol Metab 2012, 23, (10), 533-543.

22. Lee, Y. H.; Yang, H.; Lee, S. R.; Kwon, S. W.; Hong, E. J.; Lee, H. W., Welsh Onion Root (Allium fistulosum) Restores Ovarian Functions from Letrozole Induced-Polycystic Ovary Syndrome. Nutrients 2018, 10, (10).

23. Rajan, R. K.; M, S. S.; Balaji, B., Soy isoflavones exert beneficial effects on letrozole-induced rat polycystic ovary syndrome (PCOS) model through anti-androgenic mechanism. Pharmaceutical biology 2017, 55, (1), 242-251.

24. Hu, Q.; Jin, J.; Zhou, H.; Yu, D.; Qian, W.; Zhong, Y.; Zhang, J.; Tang, C.; Liu, P.; Zhou, Y.; Wang, X.; Sheng, L., Crocetin attenuates DHT-induced polycystic ovary syndrome in mice via revising kisspeptin neurons. Biomedicine \& pharmacotherapy = Biomedecine \& pharmacotherapie 2018, 107, 13631369.

25. Lu, J.; Wu, D. M.; Zheng, Y. L.; Hu, B.; Cheng, W.; Zhang, Z. F.; Li, M. Q., Troxerutin counteracts domoic acid-induced memory deficits in mice by inhibiting CCAAT/enhancer binding protein beta-mediated inflammatory response and oxidative stress. Journal of immunology 2013, 190, (7), 3466-79.

26. Baluchnejadmojarad, T.; Jamali-Raeufy, N.; Zabihnejad, S.; Rabiee, N.; Roghani, M., Troxerutin exerts neuroprotection in 6-hydroxydopamine lesion rat model of Parkinson's disease: Possible involvement of PI3K/ERbeta signaling. European journal of pharmacology 2017, 801, 72-78.

27. Zhao, H.; Liu, Y.; Zeng, J.; Li, D.; Zhang, W.; Huang, Y., Troxerutin and Cerebroprotein Hydrolysate Injection Protects Neurovascular Units from Oxygen-Glucose Deprivation and Reoxygenation-Induced Injury In Vitro. Evidence-based complementary and alternative medicine : eCAM 2018, 2018, 9859672.

28. Taylor, A. E.; McCourt, B.; Martin, K. A.; Anderson, E. J.; Adams, J. M.; Schoenfeld, D.; Hall, J. E., Determinants of abnormal gonadotropin secretion in clinically defined women with polycystic ovary syndrome. J Clin Endocrinol Metab 1997, 82, (7), 2248-2256. 
29. Sagnella, F.; Apa, R.; Guido, M.; Villa, P.; Spadoni, V.; Miceli, F.; Lanzone, A., Suppression and recovery of gonadotropin and steroid secretion by a gonadotropin-releasing hormone receptor antagonist in healthy women with normal ovulation versus women with polycystic ovary syndrome in the early follicular phase. Fertil Steril 2009, 91, (5), 1857-1863.

30. Ertunc, D.; Tok, E. C.; Savas, A.; Ozturk, I.; Dilek, S., Gonadotropin-releasing hormone antagonist use in controlled ovarian stimulation and intrauterine insemination cycles in women with polycystic ovary syndrome. Fertil Steril 2010, 93, (4), 1179-1184.

31. Kazer, R. R.; Kessel, B.; Yen, S. S., Circulating luteinizing hormone pulse frequency in women with polycystic ovary syndrome. J Clin Endocrinol Metab 1987, 65, (2), 233-236.

32. Pyun, B. J.; Yang, H.; Sohn, E.; Yu, S. Y.; Lee, D.; Jung, D. H.; Ko, B. S.; Lee, H. W., Tetragonia tetragonioides (Pall.) Kuntze Regulates Androgen Production in a Letrozole-Induced Polycystic Ovary Syndrome Model. Molecules 2018, 23, (5).

33. Jahan, S.; Abid, A.; Khalid, S.; Afsar, T.; Qurat UI, A.; Shaheen, G.; Almajwal, A.; Razak, S., Therapeutic potentials of Quercetin in management of polycystic ovarian syndrome using Letrozole induced rat model: a histological and a biochemical study. Journal of ovarian research 2018, 11, (1), 26.

34. Zhao, H.; Zhou, D.; Chen, Y.; Liu, D.; Chu, S.; Zhang, S., Beneficial effects of Heqi san on rat model of polycystic ovary syndrome through the PI3K/AKT pathway. Daru : journal of Faculty of Pharmacy, Tehran University of Medical Sciences 2017, 25, (1), 21.

35. Kabiri, N.; Tabandeh, M. R.; Tabatabaie, S. R., Beneficial effects of pioglitazone and metformin in murine model of polycystic ovaries via improvement of chemerin gene up-regulation. Daru : journal of Faculty of Pharmacy, Tehran University of Medical Sciences 2014, 22, 39.

36. Karateke, A.; Dokuyucu, R.; Dogan, H.; Ozgur, T.; Tas, Z. A.; Tutuk, O.; Agturk, G.; Tumer, C., Investigation of Therapeutic Effects of Erdosteine on Polycystic Ovary Syndrome in a Rat Model. Medical principles and practice : international journal of the Kuwait University, Health Science Centre 2018, 27, (6), 515-522.

37. Chaudhari, N. K.; Nampoothiri, L. P., Neurotransmitter alteration in a testosterone propionate-induced polycystic ovarian syndrome rat model. Hormone molecular biology and clinical investigation 2017, 29, (2), 71-77.

38. Moore, A. M.; Campbell, R. E., Polycystic ovary syndrome: Understanding the role of the brain. Frontiers in neuroendocrinology 2017, 46, 1-14.

39. Coutinho, E. A.; Kauffman, A. S., The Role of the Brain in the Pathogenesis and Physiology of Polycystic Ovary Syndrome (PCOS). Medical sciences 2019, 7, (8).

40. Shi, X.; Zhang, L.; Fu, S.; Li, N., Co-involvement of psychological and neurological abnormalities in infertility with polycystic ovarian syndrome. Arch Gynecol Obstet 2011, 284, (3), 773-778.

41. Watanabe, M.; Fukuda, A.; Nabekura, J., The role of GABA in the regulation of GnRH neurons. Frontiers in neuroscience 2014, 8, 387.

\section{Conclusions}


Taken above, these results indicated that the protection of troxerutin against PCOS partially might be due to its ability to regulate hypothalamic GABA, Glu, kisspeptin/kiss1r and NKB/NK3r. Although it remains determined whether this troxerutin-caused reversal is permanent, the present study highlighted its therapeutic potential for PCOS. More in depth research will be necessary to determine whether troxerutin targeted at hypothalamic neurotransmitters alone is a promising therapeutic approach for the treatment of PCOS.

\section{Abbreviations}

ANOVA: Analysis of Variance; ARC: arcuate nucleus; DHT: Dihydrotestosterone; ELISA: Enzyme Linked Immuno Sorbent Assay; FSH: follicle stimulating hormone; GABA: Gamma-aminobutyric acid; Glu: glutamate; GnRH: Gonadotrophin-releasing hormone; H\&E: hematoxylin and eosin; HPLC: Highperformance liquid chromatography; HPG: hypothalamus-pituitary-gonad; IGF-1:Insulin growth factor-1; IOD: integral optical density; Kiss1r: Kisspeptin 1 receptor; KNDy: kisspeptin-/ neurokinin B-/ dynorphin; LH: luteinizing hormone; ME: median eminence; NGF: nerve growth factor; NKB: neurkinin B; PCOS: Polycystic ovary syndrome;PFA: paraformaldehyde.

\section{Declarations}

\section{Acknowledgements}

We would like to thank everyone who take part in this study.

\section{Funding}

This research was supported by the funds granted by the national natural science foundation of China(No. 81571405) to Xiaonan Yan, Jiangsu Province natural science foundation of China(No. BK20161169)to Xiaonan Yan, the starting foundation for talents of xuzhou Medical University (No. D2018007) to Zixuan Gao, the Jiangsu Shuangchuang program to Zixuan Gao, Natural science research projects of Colleges and Universities in Jiangsu province(No. 19KJB310021)to Zixuan Gao.

\section{Availability of data and materials}

The datasets used and/or analyzed in the present study are available from the corresponding author on reasonable request.

\section{Authors' contributions}

$R Q Y$ and $X N Y$ conceived and designed the idea to this paper; $Z X G, X C M, Y H G, L W, P F, J$ L collected and analyzed the data, and drafted the paper; $Z A L, R Q Y$ and $X N Y$ analyzed the data and revised the final paper. All authors read and approved the final manuscript.

\section{Ethics approval and consent to participate}


The experiments were conducted in accordance with the Regulation of Animal Research Ethics in China. The ethical approval was granted by the Xuzhou Medical University Animal Research Ethics Committee (Project number 201811W011, Xuzhou, China).

\section{Consent for publication}

Not applicable.

\section{Competing interests}

The authors declare that they have no competing interests.

\section{References}

1. Goodarzi, M.O. and R. Azziz, Diagnosis, epidemiology, and genetics of the polycystic ovary syndrome. Best Pract Res Clin Endocrinol Metab, 2006. 20(2): p. 193-205.

2. Hahn, S., et al., Clinical and biochemical characterization of women with polycystic ovary syndrome in North Rhine-Westphalia. Hormone and metabolic research = Hormon- und Stoffwechselforschung = Hormones et metabolisme, 2005. 37(7): p. 438-444.

3. Carmina, E., et al., Difference in body weight between American and Italian women with polycystic ovary syndrome: influence of the diet. Human reproduction (Oxford, England), 2003. 18(11): p. 22892293.

4. Zumoff, B., et al., A chronobiologic abnormality in luteinizing hormone secretion in teenage girls with the polycystic-ovary syndrome. The New England journal of medicine, 1983. 309(20): p. 1206-1209.

5. Gilling-Smith, C., et al., Evidence for a primary abnormality of thecal cell steroidogenesis in the polycystic ovary syndrome. Clinical endocrinology, 1997. 47(1): p. 93-99.

6. Tsutsumi, R. and N.J.G. Webster, GnRH pulsatility, the pituitary response and reproductive dysfunction. Endocrine journal, 2009. 56(6): p. 729-737.

7. Kaiser, U.B., P.M. Conn, and W.W. Chin, Studies of gonadotropin-releasing hormone (GnRH) action using GnRH receptor-expressing pituitary cell lines. Endocrine reviews, 1997. 18(1): p. 46-70.

8. Smith, M.J. and L. Jennes, Neural signals that regulate GnRH neurones directly during the oestrous cycle. Reproduction (Cambridge, England), 2001. 122(1): p. 1-10.

9. Chaudhari, N., M. Dawalbhakta, and L. Nampoothiri, GnRH dysregulation in polycystic ovarian syndrome (PCOS) is a manifestation of an altered neurotransmitter profile. Reproductive biology and endocrinology : RB\&E, 2018. 16(1): p. 37-37.

10. de Roux, N., et al., Hypogonadotropic hypogonadism due to loss of function of the KiSS1-derived peptide receptor GPR54. Proceedings of the National Academy of Sciences of the United States of America, 2003. 100(19): p. 10972-10976.

11. Topaloglu, A.K., et al., TAC3 and TACR3 mutations in familial hypogonadotropic hypogonadism reveal a key role for Neurokinin B in the central control of reproduction. Nature genetics, 2009. 41(3): 
p. 354-358.

12. Goodman, R.L., et al., Kisspeptin neurons in the arcuate nucleus of the ewe express both dynorphin $A$ and neurokinin B. Endocrinology, 2007. 148(12): p. 5752-5760.

13. Foradori, C.D., et al., Colocalisation of dynorphin a and neurokinin B immunoreactivity in the arcuate nucleus and median eminence of the sheep. Journal of neuroendocrinology, 2006. 18(7): p. 534-541.

14. Messager, S., et al., Kisspeptin directly stimulates gonadotropin-releasing hormone release via $G$ protein-coupled receptor 54. Proceedings of the National Academy of Sciences of the United States of America, 2005. 102(5): p. 1761-1766.

15. Krajewski, S.J., et al., Morphologic evidence that neurokinin B modulates gonadotropin-releasing hormone secretion via neurokinin 3 receptors in the rat median eminence. The Journal of comparative neurology, 2005. 489(3): p. 372-386.

16. Panat, N.A., et al., Troxerutin, a plant flavonoid, protects cells against oxidative stress-induced cell death through radical scavenging mechanism. Food chemistry, 2016. 194: p. 32-45.

17. Zhang, Z.f., et al., Troxerutin protects the mouse liver against oxidative stress-mediated injury induced by D-galactose. Journal of agricultural and food chemistry, 2009. 57(17): p. 7731-7736.

18. Lu, J., et al., Troxerutin protects against high cholesterol-induced cognitive deficits in mice. Brain : a journal of neurology, 2011. 134(Pt 3): p. 783-797.

19. Farajdokht, F., et al., Troxerutin protects hippocampal neurons against amyloid beta-induced oxidative stress and apoptosis. EXCLI journal, 2017. 16: p. 1081-1089.

20. Lu, J., et al., Troxerutin counteracts domoic acid-induced memory deficits in mice by inhibiting CCAAT/enhancer binding protein $\beta$-mediated inflammatory response and oxidative stress. J Immunol, 2013. 190(7): p. 3466-79.

21. Baluchnejadmojarad, T., et al., Troxerutin exerts neuroprotection in 6-hydroxydopamine lesion rat model of Parkinson's disease: Possible involvement of PI3K/ERbeta signaling. Eur J Pharmacol, 2017. 801: p. 72-78.

22. Hoseindoost, M., et al., Effects of troxerutin on inflammatory cytokines and BDNF levels in male offspring of high-fat diet fed rats. Avicenna J Phytomed, 2019. 9(6): p. 597-605.

23. Lu, J., et al., NGF-Dependent activation of TrkA pathway: A mechanism for the neuroprotective effect of troxerutin in D-galactose-treated mice. Brain Pathol, 2010. 20(5): p. 952-65.

24. Palomba, S., A. Falbo, and F. Zullo, Management strategies for ovulation induction in women with polycystic ovary syndrome and known clomifene citrate resistance. Current opinion in obstetrics \& gynecology, 2009. 21(6): p. 465-473.

25. Misso, M.L., et al., Status of clomiphene citrate and metformin for infertility in PCOS. Trends in endocrinology and metabolism: TEM, 2012. 23(10): p. 533-543.

26. Lee, Y.H., et al., Welsh Onion Root (Allium fistulosum) Restores Ovarian Functions from Letrozole Induced-Polycystic Ovary Syndrome. Nutrients, 2018. 10(10). 
27. Rajan, R.K., S.S. M, and B. Balaji, Soy isoflavones exert beneficial effects on letrozole-induced rat polycystic ovary syndrome (PCOS) model through anti-androgenic mechanism. Pharm Biol, 2017. 55(1): p. 242-251.

28. Hu, Q., et al., Crocetin attenuates DHT-induced polycystic ovary syndrome in mice via revising kisspeptin neurons. Biomed Pharmacother, 2018. 107: p. 1363-1369.

29. Zhao, H., et al., Troxerutin and Cerebroprotein Hydrolysate Injection Protects Neurovascular Units from Oxygen-Glucose Deprivation and Reoxygenation-Induced Injury In Vitro. Evid Based Complement Alternat Med, 2018. 2018: p. 9859672.

30. Taylor, A.E., et al., Determinants of abnormal gonadotropin secretion in clinically defined women with polycystic ovary syndrome. The Journal of clinical endocrinology and metabolism, 1997. 82(7): p. 2248-2256.

31. Sagnella, F., et al., Suppression and recovery of gonadotropin and steroid secretion by a gonadotropin-releasing hormone receptor antagonist in healthy women with normal ovulation versus women with polycystic ovary syndrome in the early follicular phase. Fertility and sterility, 2009. 91(5): p. 1857-1863.

32. Ertunc, D., et al., Gonadotropin-releasing hormone antagonist use in controlled ovarian stimulation and intrauterine insemination cycles in women with polycystic ovary syndrome. Fertility and sterility, 2010. 93(4): p. 1179-1184.

33. Kazer, R.R., B. Kessel, and S.S. Yen, Circulating luteinizing hormone pulse frequency in women with polycystic ovary syndrome. The Journal of clinical endocrinology and metabolism, 1987. 65(2): p. 233-236.

34. Pyun, B.J., et al., Tetragonia tetragonioides (Pall.) Kuntze Regulates Androgen Production in a Letrozole-Induced Polycystic Ovary Syndrome Model. Molecules, 2018. 23(5).

35. Jahan, S., et al., Therapeutic potentials of Quercetin in management of polycystic ovarian syndrome using Letrozole induced rat model: a histological and a biochemical study. J Ovarian Res, 2018. 11(1): p. 26.

36. Zhao, H., et al., Beneficial effects of Heqi san on rat model of polycystic ovary syndrome through the PI3K/AKT pathway. Daru, 2017. 25(1): p. 21.

37. Kabiri, N., M.R. Tabandeh, and S.R. Tabatabaie, Beneficial effects of pioglitazone and metformin in murine model of polycystic ovaries via improvement of chemerin gene up-regulation. Daru, 2014. 22 : p. 39.

38. Karateke, A., et al., Investigation of Therapeutic Effects of Erdosteine on Polycystic Ovary Syndrome in a Rat Model. Med Princ Pract, 2018. 27(6): p. 515-522.

39. Chaudhari, N.K. and L.P. Nampoothiri, Neurotransmitter alteration in a testosterone propionateinduced polycystic ovarian syndrome rat model. Hormone molecular biology and clinical investigation, 2017. 29(2): p. 71-77.

40. Moore, A.M. and R.E. Campbell, Polycystic ovary syndrome: Understanding the role of the brain. Front Neuroendocrinol, 2017. 46: p. 1-14. 
41. Coutinho, E.A. and A.S. Kauffman, The Role of the Brain in the Pathogenesis and Physiology of Polycystic Ovary Syndrome (PCOS). Med Sci (Basel), 2019. 7(8).

42. Shi, X., et al., Co-involvement of psychological and neurological abnormalities in infertility with polycystic ovarian syndrome. Archives of gynecology and obstetrics, 2011. 284(3): p. 773-778.

43. Watanabe, M., A. Fukuda, and J. Nabekura, The role of GABA in the regulation of GnRH neurons. Front Neurosci, 2014. 8: p. 387.

\section{Figures}

a
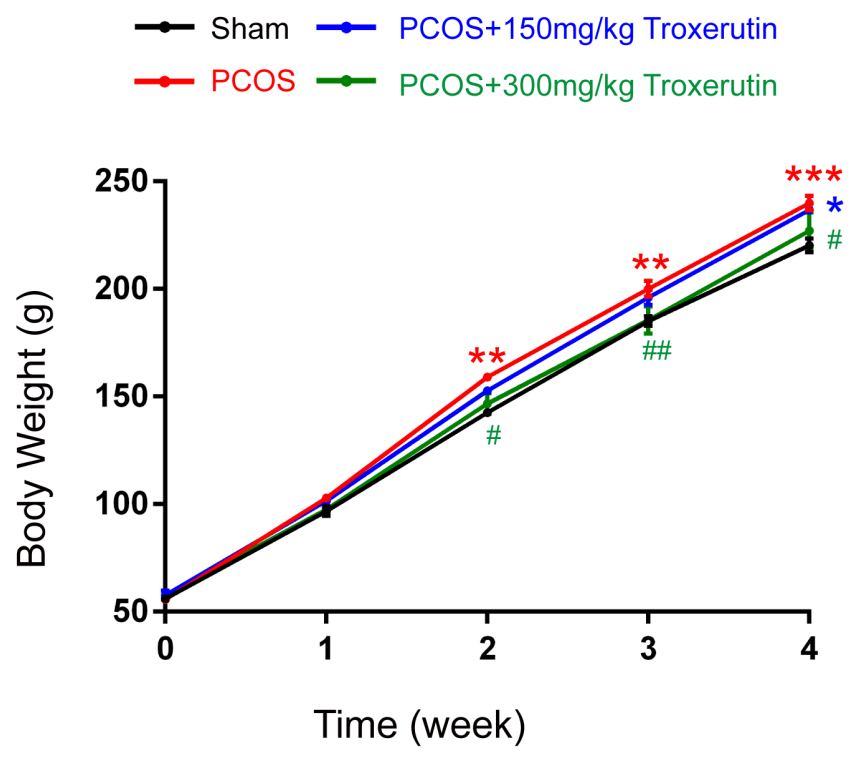

b
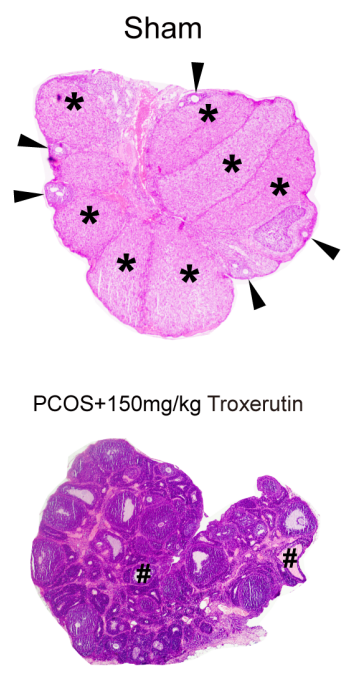

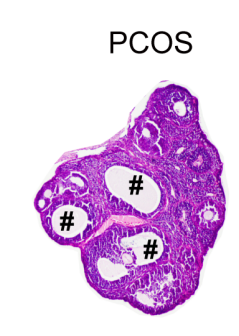

PCOS+300mg/kg Troxerutin

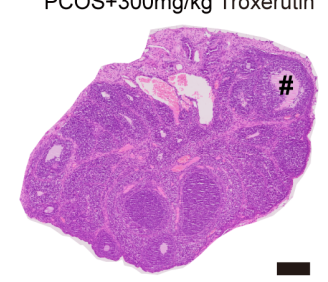

\section{Figure 1}

Troxerutin reduced obesity and improved abnormal ovarian morphology in PCOS rats (a) Body weight (b) Hematoxylin and eosin staining of representative ovaries. For a, $\mathrm{P}$ values were determined by one-way ANOVA with Turkey's multiple comparison test and data were presented as means \pm s.e.m. $n=12$ rats per

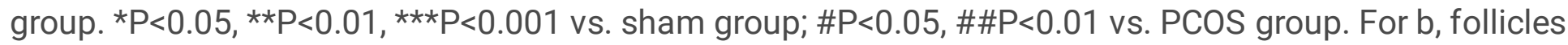
in various stages of development were indicated by black triangles, corpora lutea were indicated by asterisks, while the cystic follicles were indicated by hashtags. Scale bar: $200 \mu \mathrm{m}$. Images were representative of three independent experiments with similar results. 
a

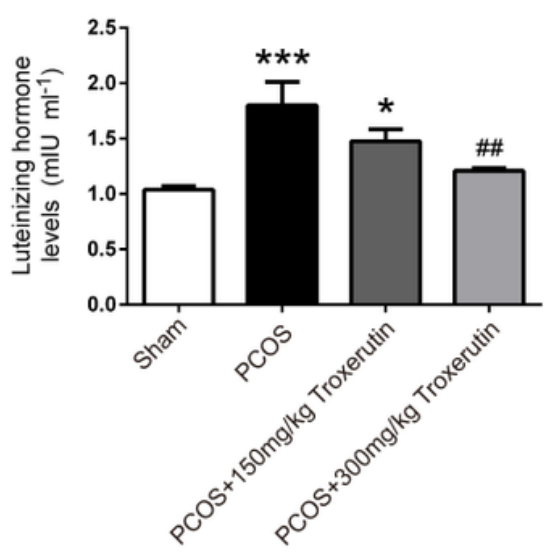

b

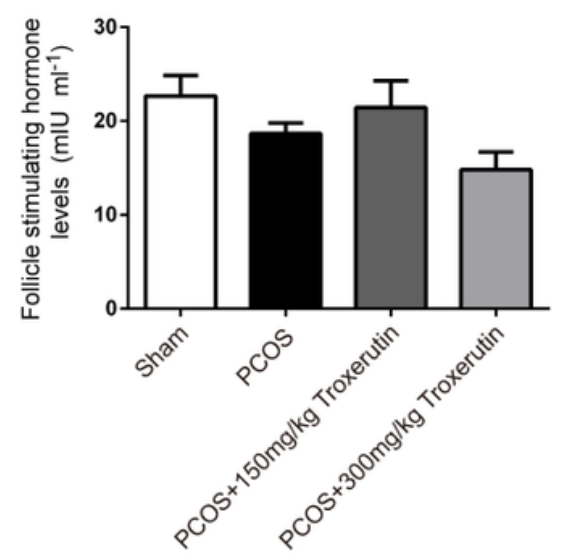

C

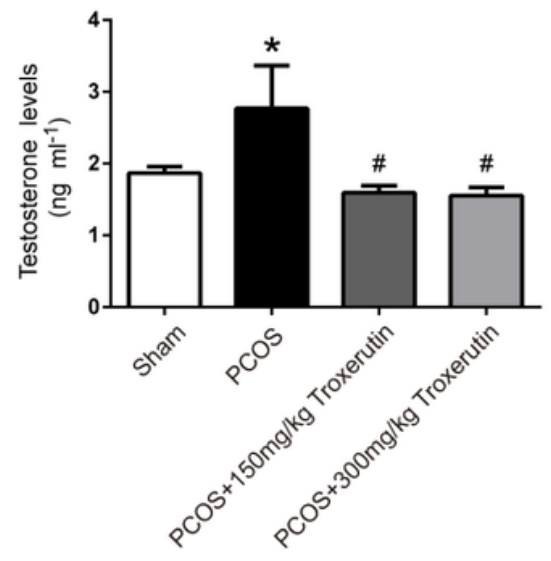

\section{Figure 2}

Troxerutin reversed the abnormal serum levels of gonadotrophin and testosterone in PCOS rats (a) Serum LH levels (b) Serum FSH levels (c) Serum testosterone levels. P values were determined by one-way ANOVA with Turkey's multiple comparison test and data were presented as means \pm s.e.m. $n=9$ rats per group. ${ }^{*} \mathrm{P}<0.05$, ${ }^{* \star *} \mathrm{P}<0.001$ vs. sham group; $\# \mathrm{P}<0.05$, \#\#P<0.01 vs. $\mathrm{PCOS}$ group.

a

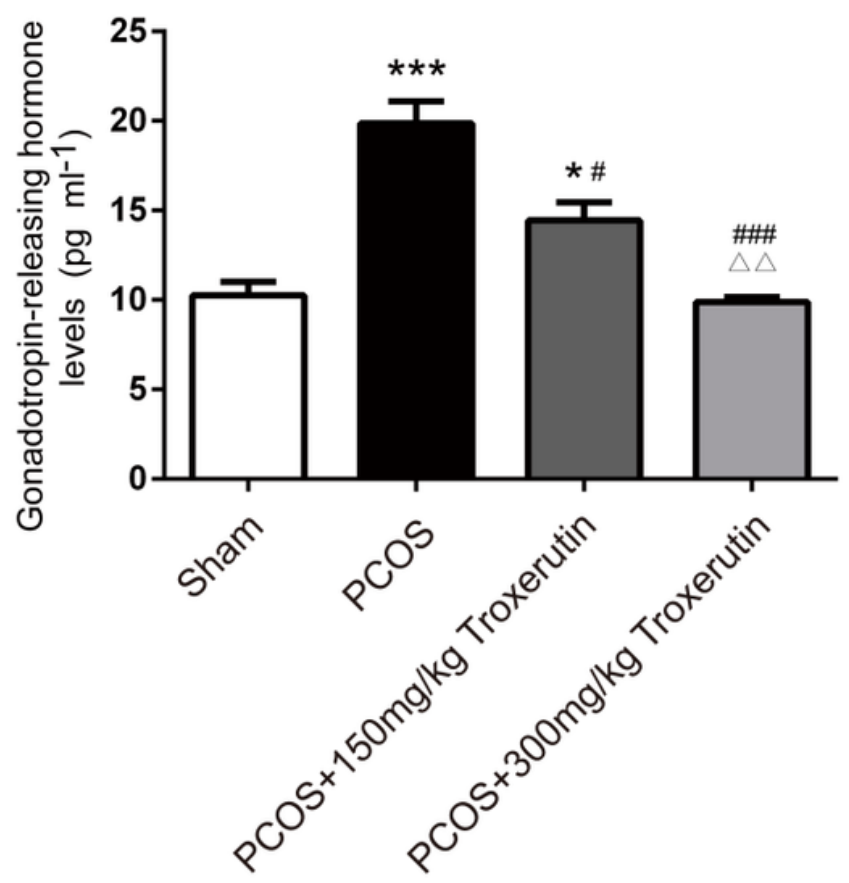

b
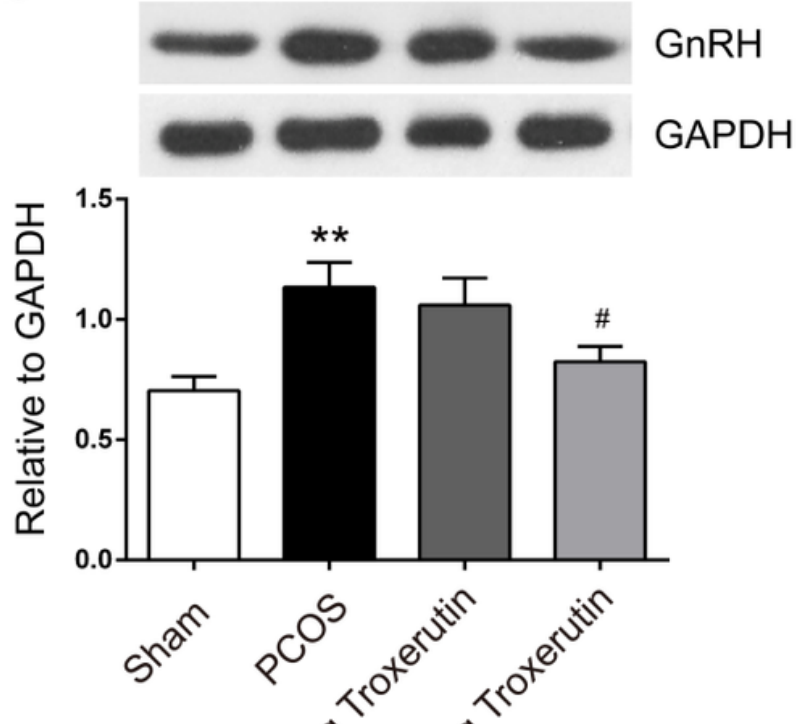

Figure 3 
Troxerutin reduced the elevated GnRH levels in PCOS rats (a) Serum levels of GnRH (b) Hypothalamic status of GnRH. P values were determined by one-way ANOVA with Turkey's multiple comparison test and data were presented as means \pm s.e.m. $n=9$ rats per group. ${ }^{*} P<0.05,{ }^{\star \star} P<0.01,{ }^{\star \star \star} P<0.001$ vs. sham group; $\# P<0.05, \# \# P<0.01$ vs. $P C O S$ group; $\triangle \triangle P<0.01$ vs. $P C O S+150 \mathrm{mg} / \mathrm{kg}$ troxerutin group.

a

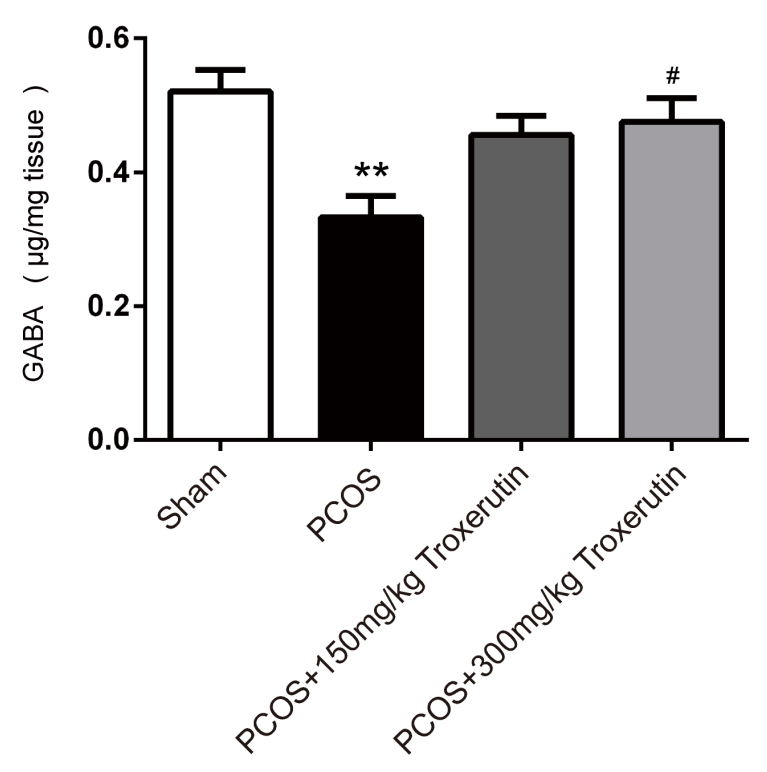

b

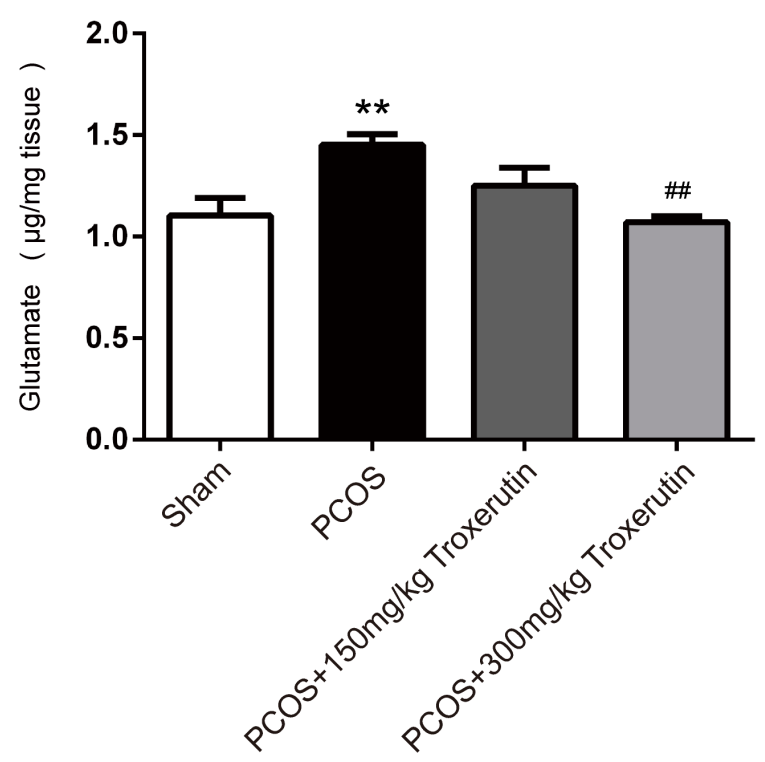

Figure 4

Troxerutin altered GnRH regulatory neurotransmitters in the hypothalamus of PCOS rats (a) Hypothalamic levels of GnRH inhibitory GABA (b) Hypothalamic levels of GnRH stimulatory glutamate. P values were determined by one-way ANOVA with Turkey's multiple comparison test and data were presented as means \pm s.e.m. $n=9$ rats per group. ${ }^{*} \mathrm{P}<0.01$ vs. sham group; $\# \mathrm{P}<0.05, \# \# P<0.01$ vs. $\mathrm{PCOS}$ group. 


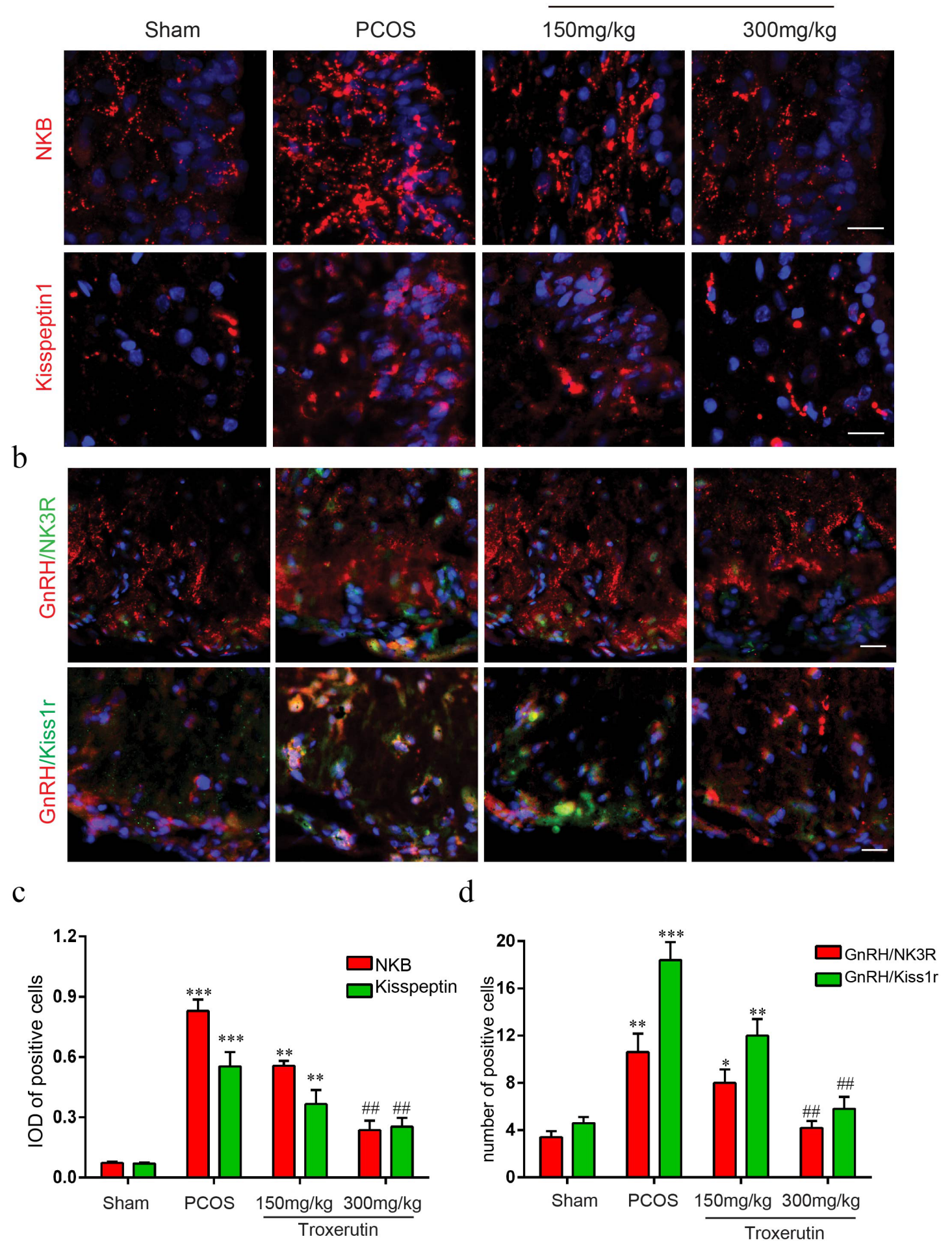

\section{Figure 5}

Troxerutin reduced the expression of Kisspepetin1/Kiss1 $r$ and Neurokinin B/NK3r in the hypothalamus (a) and (b) Immunofluorescence photomicrograph showing that the expression of Kisspeptin 1 and NKB in the arcuate nucleus (ARC) and Kiss1r, NK3r in GnRH positive neurons in the median eminence (ME). Scale

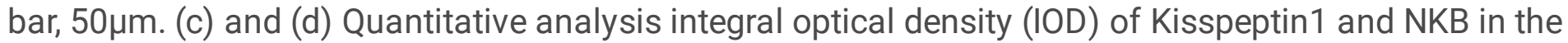
ARC and the number of Kiss1r/ GnRH and NK3r/GnRH positive cells in the ME. P values were determined 
by one-way ANOVA with Turkey's multiple comparison test and data were presented as means \pm s.e.m. $n=6$ rats per group. ${ }^{\star \star} P<0.01,{ }^{\star \star *} P<0.001$ vs. sham group; $\# P<0.05$, \#\#P<0.01 vs. PCOS group. 